\title{
APLICAÇÃO DE TÉCNICAS PARA ESTUDOS DE INGESTÃO, COMPOSIÇÃO DA DIETA E DIGESTIBILIDADE
}

\section{(Application of techniques for intake, diet composition and digestibility studies)}

\author{
BERCHIELLI, T.T.'; OLIVEIRA, S.G. ${ }^{2}$; GARCIA, A.V. ${ }^{3}$ \\ 'Departamento de Zootecnia- FCAV/UNESP - Jaboticabal, SP, Pesquisadora do CNPq, \\ ttberchi@fcav.unesp.br; \\ 2Programa de Pós-graduação em Zootecnia, FCAV/UNESP - Jaboticabal, SP; \\ ${ }^{3}$ Facultade de Veterinaria, Universidad de Zaragoza - Zaragoza - Espanha.
}

RESUMO - A adequada nutrição dos animais de produção é dependente do conhecimento das exigências dos animais e valor nutritivo dos alimentos. No entanto, em muitas situações, a obtenção das informações necessárias não é possível ou é dificultada pela falta de recursos ou alto custo dos procedimentos experimentais, sendo necessária a aplicação de técnicas que possam estimar de forma precisa os parâmetros desejados. A avaliação de animais em pastagem é bastante dificultada pela impossibilidade em se controlar a ingestão e excreção dos animais, onde a aplicação de modelos matemáticos e a utilização de indicadores surgem como alternativas que podem reduzir custos e aumentar a precisão das estimativas. O objetivo dessa revisão foi apresentar as principais técnicas em uso que visam determinar os parâmetros consumo, digestibilidade e composição da dieta em diferentes sistemas de produção assim como as particularidades, vantagens e desvantagens dos métodos empregados. A escolha da técnica empregada depende fundamentalmente dos objetivos a serem alcançados, dos recursos disponíveis e da precisão necessária. A utilização de indicadores é uma ferramenta bastante apropriada, possibilitando a estimativa de consumo e digestibilidade nos diferentes compartimentos gastrintestinais. O emprego dos alcanos tem apresentado resultados bastante satisfatórios na avaliação da composição das dietas, em especial em sistemas de pastagem.

Palavras-chave: Avaliação de alimentos, consumo, digestibilidade, indicadores.

ABSTRACT - The proper nutrition of food production animals primarily depends on the precise knowledge of animal requirements and of the nutritional values of the feed. However, under many circumstances, it is not possible to obtain the needed information due to lack of resources or to the high cost of the experimental procedures, and therefore, techniques need to be applied in order to accurately estimate the desired parameters. The evaluation of grazing animals on pastures is impaired by the unfeasibility of controlling intake and excretion, and here the application of mathematical models and the use of marker become an alternative to reduce costs and to improve the accuracy of the estimates. This revision aimed at presenting the main techniques applied for determining the parameters intake, digestibility and diet composition in different production systems, as well as the peculiarities, advantages, and disadvantages of the employed methods. The choice of a technique primarily depends on the objectives of the study, on the available resources, and on the required accuracy. The use of markers is an appropriate tool, allowing to estimate intake and digestibility in different gastrointestinal compartments. The use of alkanes has presented good results for the evaluation of diet composition, particularly in grazing systems.

Key-words: Evaluation of feeds, intake, digestibility, markers. 


\section{Introdução}

O valor nutritivo de um alimento dependerá, fundamentalmente, da quantidade de nutrientes que é destinado ao animal, do consumo e da digestibilidade dos mesmos. Um dos fatores de maior influência sobre a resposta animal em produção é a quantidade total de nutrientes absorvidos da dieta, sendo a ingestão e a digestibilidade parâmetros chaves em qualquer sistema de avaliação de alimentos.

MERTENS (1994) cita que o desempenho animal é função do consumo de nutrientes digestíveis e metabolizáveis, uma vez que cerca de 60 a $90 \%$ das variações em desempenho são explicadas pelas variações correspondentes ao consumo e 10 a 40 \% são creditadas a variações obtidas com a digestibilidade. A determinação da ingestão e digestibilidade é facilitada quando se trabalha com animais em confinamento, tornando-se mais difícil obter tais determinações quando se avaliam animais em sistema de pastagem (POPPI et al., 2000).

O objetivo dessa revisão é apresentar as principais técnicas em uso que visam determinar os parâmetros consumo, digestibilidade e composição da dieta em diferentes sistemas de produção assim como as particularidades, vantagens e desvantagens dos métodos empregados.

\section{Estudos sobre a Ingestão}

As teorias que explicam o controle do consumo voluntário em ruminantes admitem ser este mecanismo um produto da ação integrada ou isolada de fatores físicos (saciedade física) e fisiológicos (saciedade química) (ALLEN, 2000). A demanda energética do animal define o consumo de dietas de alta densidade calórica, ao passo que a capacidade física do trato gastrintestinal determina o consumo de dietas com baixo valor nutritivo (VAN SOEST, 1994).

Segundo HOVEL et al. (1986), a ingestão voluntária de alimentos de baixa qualidade ou com elevados teores de fibra em detergente neutro (FDN) é mais correlacionada com o potencial de degradação e à degradabilidade efetiva da matéria seca do que com a digestibilidade in vivo, concluindo que as características de degradação das forragens podem ser úteis na predição.

A oferta do alimento em regime de confinamento pode ser fixa, ad libitum (alimento fornecido a vontade para os animais) ou em níveis variados. O nível fixo, geralmente, é utilizado quando se deseja medir a digestibilidade da dieta a um nível de consumo próximo ao da mantença. O regime de alimentação em diferentes níveis é recomendado para a avaliação de forrageiras tropicais, quando níveis crescentes de oferta resultam em aumento do consumo, principalmente, devido a possibilidade de dar maior oportunidade de seleção a dieta aos animais. Contudo, o mais utilizado é o fornecimento ad libitum, que se obtém fornecendo 15 a $20 \%$ a mais de matéria seca sobre o consumo máximo observado (LASCANO et al., 1990). Esta metodologia é bastante simples, baseando-se na diferença entre o fornecido e o alimento recusado pelos animais (sobras).

Em relação aos animais em regime de pasto, existem metodologias diretas e indiretas, para medir consumo do animal a pasto. O consumo diário de matéria seca de animais em pastagem pode ser medido diretamente pelo monitoramento do comportamento de pastejo ou diretamente, por meio da pesagem dos animais, antes e após cada pastejo, sendo que a redução da forragem decorrente do pastejo dividida pelo número de animais e dias de pastejo fornece uma estimativa do consumo diário. Neste caso, a eficiência é limitada por possíveis erros nas estimativas de produção de forragem inicial e final e pelo crescimento ou perda de plantas durante o pastejo (MINSON, 1990).

Deve-se levar em consideração que durante o período de pastejo a biomassa cresce, e o uso de gaiolas de exclusão para determinar esse crescimento se faz necessário. Entre os principais inconvenientes da técnica, destaca-se que: 1 - o crescimento da biomassa nas gaiolas de exclusão pode não ser o mesmo que fora dela, 2 - não se considera a variabilidade individual no consumo e, finalmente, 3 - o que se observa é o desaparecimento total da biomassa, não fornecendo informações sobre a seleção 
individual que os animais realizam sobre esta.

Há necessidade de correções para as possíveis perdas (respiração, defecação e micção) e ganhos (ingestão de minerais, de água, de solo) não relacionados com a forragem ingerida. Um outro aspecto negativo da técnica é considerar o consumo de material fresco e não da matéria seca, uma vez que não se obtém a composição química do material pastejado.

A avaliação pelo comportamento animal requer estimativas do tempo de pastejo, número, tamanho ou peso dos bocados. Esta metodologia apresenta vantagens pelo reduzido estresse provocado aos animais durante o experimento, facilidade de coleta de dados e aplicabilidade em diferentes condições da pastagem.

Diante dos importantes inconvenientes apresentados pelos métodos descritos para o cálculo da ingestão de animais em pastagem, MAYES et al. (1986) propuseram a utilização dos alcanos presentes nas ceras da cutícula como indicadores para determinação de tal parâmetro, devendo-se considerar que o tamanho de cadeia de carbonos dos alcanos utilizados como indicadores está normalmente entre 25 e 35 carbonos. Os n-alcanos presentes nas plantas são normalmente de cadeia ímpar, sendo os de número par de carbonos muito raros nas plantas superiores. As recuperações fecais de alcanos que se diferenciam apenas em um átomo de carbono $\left(\mathrm{C}_{32}\right.$ e $\mathrm{C}_{33}$, por exemplo), tendem a ser muito similares. Embora este ponto não seja estritamente certo, em linhas gerais pode ser considerado válido.

Tendo-se em conta estes critérios, a ingestão (I) de um indicador externo $\left(C_{32}\right.$, por exemplo) será igual à dose administrada $(D)$ somada a quantidade ingerida com o alimento, representada pela quantidade de matéria seca ingerida $(F)$ multiplicada pela concentração do indicador $\left(F_{32}\right)$, o que é o mesmo:

$$
I_{32}=D_{32}+\left(F \times F_{32}\right)
$$

A partir daqui, pode-se calcular um pool fecal teórico de distribuição do indicador a partir da igualdade:

$$
H \times H_{32}=D_{32}+\left(F \times F_{32}\right)
$$

Onde, $H_{32}$ é a concentração de $\mathrm{C}_{32}$ nas fezes, e Ho pool fecal teórico de distribuição. Observase que este "pool" fecal teórico somente coincidirá com a produção fecal real quando a recuperação do indicador for $100 \%$.

Assumindo que a recuperação de um alcano natural próximo $\mathrm{a} \mathrm{C}_{32}\left(\mathrm{C}_{33}\right.$, por exemplo) é igual a:

$$
H \times H_{33}=F \times F_{33}
$$

e

$$
\frac{D_{32}+\left(F \times F_{32}\right)}{H_{32}} \times H_{33}=F \times F_{33}
$$

onde,

$$
F=\frac{D_{32} \times H_{33}}{F_{33} \times H_{32}-F_{32} \times H_{33}}
$$

e

$$
F=\frac{D_{32}}{F_{33} x \frac{H_{32}}{H_{33}}-F_{32}}
$$

Pode-se estimar a ingestão sem a necessidade de conhecer a recuperação do indicador, sempre que se administrar um indicador externo. O problema consiste em que o método somente é aplicável quando os animais consomem dietas com apenas um ingrediente, pois caso contrário $F_{32}$ e $F_{33}$ dependerão da seleção que o animal exerça, e da concentração dos alcanos considerados em cada um dos componentes da dieta. É evidente, portanto, a necessidade de conhecer a composição da dieta selecionada pelos animais quando a oferta não se reduz a apenas um componente.

\section{Estudo da Composição da Dieta}

As determinações dos componentes da dieta que os animais ingerem podem ser realizadas através dos métodos de avaliação de pastagem 
ou por estimativas com animais. Como a forragem amostrada pelo método de corte não representa corretamente a dieta selecionada pelo animal, utiliza-se animais fistulados no esôfago ou a técnica de simulação de pastejo para a obtenção das amostras utilizadas no ensaio de digestibilidade, o qual pode ser in vitro (POND et al., 1989), in situ (NOCEK, 1988) ou pelo método de produção de gás in vitro (MALAFAIA et al., 1996).

Animais fistulados no esôfago e técnicas micro-histológicas: Baseada nas amostras obtidas de forragens em animais fistulados no esôfago e análises micro-histológicas das mesmas. Segundo MAYES e DOVE (2000) é um método que apresenta sérios inconvenientes pelas possíveis variações. Os animais fistulados no esôfago podem ser de raça e estado fisiológico distinto dos animais experimentais e são mantidos em jejum durante o dia anterior ao período de amostragem, além do fato que o período de pastejo é realizado em intervalos muito curtos comparados com o tempo normal do pastejo. Todos estes fatores levantam sérias dúvidas sobre a capacidade de seleção dos animais fistulados no esôfago, considerando ainda que pode haver contaminação com a saliva e o fato da mastigação produzir partículas muito pequenas que podem atravessar o esôfago com a fase líquida sem ser exteriorizada com a amostra. Por outro lado, a análise micro-histológica da amostra é tediosa e consome muito tempo, mostrando desta forma não ser aplicável como técnica de rotina em experimentos com um grande número de amostras.

Comportamento animal: A estimativa da composição da dieta pode também ser obtida a partir de um estudo do tempo de pastejo, determinando a proporção de tempo que os animais investem em consumir cada espécie presente no pasto, o número de bocados, ou seja, o número de vezes que o animal realiza a preensão de alimento, por unidade de tempo que os animais destinam a cada espécie e o tamanho desses bocados. No entanto, quando há necessidade de estudar o comportamento individualizado de um número maior de animais, apresenta o inconveniente da falta de constância, tanto do número de bocados dedicado a cada espécie vegetal, como do seu tamanho.

Métodos dos $n$-alcanos: Com base na premissa de que as proporções dos diferentes alcanos na dieta se mantêm nas fezes, considerando uma dieta constituída por três componentes:

$$
\sum\left[\frac{H i}{H t}-\frac{x A i+y B i+z C i}{x A t+y B t+z C t}\right]_{a l c: 1 \ldots n}^{2}
$$

onde:

$x=$ proporção do componente A na dieta;

$y=$ proporção do componente B na dieta;

$z=1-x-y$ proporção do componente $C$ na dieta;

$A i=$ concentração do alcano i no componente $\mathrm{A}$;

$B i=$ concentração do alcano i no componente $B$;

$C i=$ concentração do alcano i no componente $C$;

$A t=$ concentração total de alcanos no componente $A$;

$B t=$ concentração total de alcanos no componente $B$;

$C t=$ concentração total de alcanos no componente $C$;

$H i=$ concentração nas fezes do alcano il recuperação fecal;

$H t=$ concentração total de alcanos nas fezes/ recuperação fecal.

Em condições ideais, estes desvios ao quadrado deveriam ser igual a zero; no entanto, na prática, o que se pretende é minimizar seu valor. DOVE e MAYES (1991) têm demonstrado uma série de considerações que há de se ter em conta antes de aplicar esta metodologia

Primeiramente, quanto maiores forem as diferenças entre espécies na composição de n-alcanos, maior será a sensibilidade da estimativa da composição da dieta; todavia quando uma espécie apresenta baixos níveis de n-alcanos em relação às outras, será estimada com baixa sensibilidade relativa. Portanto, a maior sensibilidade será obtida com espécies com conteúdo total de n-alcanos semelhantes, porém com padrões marcadamente diferentes. $E$, em segundo lugar, o maior número de espécies que podem ser identificadas depende do número de alcanos usados nas análises, entretanto esta 
Aplicação de técnicas para estudos de ingestão, composição da dieta e digestibilidade

circunstância depende do método matemático utilizado.

O método dos alcanos tem sido testado com êxito em estudos com combinações de uma gramínea e uma leguminosa de clima temperado, para estimar as proporções de folha, caule e espiga da planta inteira de cevada consumida por ovinos ou para estimar as proporções de cevada, núcleo protéico e palha que consomem cordeiros em crescimento alimentados com dietas concentradas. Todavia, não existem estudos em animais consumindo forragens tropicais, apesar da importância dos sistemas extensivos em determinados países (MAYES et al., 1995).

Os principais inconvenientes do método consistem em: comportamento dos animais em confinamento não é igual ao em pastejo, logo os estudos em gaiolas de metabolismo não têm porque serem extrapolados para o campo; é preciso obter amostras absolutamente representativas do que o animal ingere, e isto nem sempre é fácil; decorrente do fato de que a administração de alcanos se realiza uma vez ao dia, podem ocorrer variações importantes na concentração fecal; a preparação dos pelets e a análise das amostras requerem métodos complexos; é imprescindível desenvolver métodos que permitam estimar uma recuperação fecal confiável dos diferentes alcanos em condições de pastejo.

\section{Estudos de Digestibilidade}

As técnicas para avaliação da digestibilidade dos alimentos possivelmente são as mais conhecidas e fundamentadas, sendo este assunto já amplamente discutido na literatura (STERN et al., 1997; TITGEMEYER, 1997; ZEOULA et al., 2000, ÍTAVO et al., 2002). A escolha da metodologia para determinação da digestibilidade nos diferentes compartimentos do trato gastrintestinal é dependente de que compartimento se deseja obter informações, além dos aspectos operacionais e econômicos do processo. Em muitos casos a determinação direta não é possível, como quando se dejesa estimar a digestibilidade ruminal da matéria seca e nutriente, devendo-se lançar mão de técnicas que viabilizem sua estimativa.
Método in vivo: Considerados como os mais confiáveis, são baseados na observação das quantidades ingeridas e excretadas das frações de interesse no alimento.

Digestibilidade $=\frac{\text { Ingerido }- \text { Excretado }}{\text { Ingerido }}=1-\frac{E}{I}$

No entanto, apresenta o inconveniente de requerer maior número de animais, controle rigoroso da quantidade ingerida e equipamentos, como gaiolas metabólicas, que possibilitem a coleta da excreta dos animais.

Em situações onde não é possível o controle da ingestão e ou excreção dos animais a utilização de indicadores é uma importante ferramenta a ser adotada, apesar dos inconvenientes apresentados com o uso da metodologia.

COCHRAN et al. (1986) relatam que a fibra em detergente neutro (FDN) e a fibra em detergente ácido (FDA) indigestíveis têm apresentado bons resultados quanto à recuperação nas fezes em algumas forragens. Uma das razões para a variabilidade nos resultados de digestibilidade obtidos com FDN e FDA indigestíveis está associada ao o método de determinação e com processo de secagem das amostras. VAN SOEST (1965) cita a importância com os cuidados no processo de secagem das amostras, onde em temperaturas superiores a $60^{\circ} \mathrm{C}$ pode favorecer a ocorrência da reação de Maillard, formando compostos indigestíveis resultantes da complexação entre a fração protéica e carboidratos solúveis. A determinação laboratorial da fração indigestível pode englobar estes compostos causando uma superestimativa dos resultados. Em trabalho realizado por BERCHIELLI et al. (2005), observa-se grande variabilidade na recuperação dos indicadores na avaliação de diferentes volumosos, mostrando que apenas o FDN in vitro e FDA in situ para o feno de Tifton, o FDA in vitro para a silagem de milho e o cromo para a cana não diferiram de $100 \%$ de recuperação (TABELA 1). 
TABELA 1 - RECUPERAÇÃO PERCENTUAL DOS MARCADORES INTERNOS E EXTERNO NAS FEZES (\%) DE DIFERENTES VOLUMOSOS.

\begin{tabular}{lcccccc}
\hline \multirow{2}{*}{ Volumosos } & \multicolumn{5}{c}{ Marcadores } & \multirow{2}{*}{ CV (\%) } \\
\cline { 2 - 6 } & FDNis & FDNiv & FDAis & FDAiv & Cromo & \\
\hline Feno Tifton & $127,08^{*}$ & 101,78 & 94,08 & $72,53^{*}$ & $142,73^{*}$ & 22,28 \\
Sil. Milho & $131,39^{*}$ & $148,12^{*}$ & $47,26^{*}$ & 108,38 & $159,18^{*}$ & 18,89 \\
Cana & $173,29^{*}$ & $145,20^{*}$ & $153,78^{*}$ & $229,90^{*}$ & 75,29 & 23,66 \\
\hline
\end{tabular}

Fibra em detergente neutro (FDNis) e ácido (FDAis) indigestíveis incubados in situ; Fibra em detergente neutro (FDNiv) e ácido indigestível (FDAiv) incubados in vitro.

CV - Coeficiente de variação.

Valores diferentes de 100 a $5 \%$ pelo teste de Tukey.

Fonte: BERCHIELLI et al. (2005).

TABELA 2 - TEORES DOS INDICADORES FDNi E FDAi EM AMOSTRAS DA DIETA, DIGESTA DUODENAL E FEZES.

\begin{tabular}{|c|c|c|c|c|c|c|}
\hline \multirow{3}{*}{ Tratamentos } & \multicolumn{6}{|c|}{ Teores dos indicadores (\%) } \\
\hline & \multicolumn{3}{|c|}{ FDNi } & \multicolumn{3}{|c|}{ FDAi } \\
\hline & Dieta & Duodeno & Fezes & Dieta & Duodeno & Fezes \\
\hline 144SL & 29,13 & 26,24 & 40,42 & 8,26 & 9,81 & 12,99 \\
\hline $144 C L$ & 27,43 & 25,80 & 39,91 & 7,76 & 9,58 & 13,03 \\
\hline 288SL & 27,04 & 25,88 & 39,85 & 8,45 & 10,15 & 12,57 \\
\hline $288 \mathrm{CL}$ & 28,24 & 26,47 & 40,71 & 7,83 & 9,94 & 13,24 \\
\hline \multirow[t]{2}{*}{ CV (\%) } & 42,71 & 19,60 & 14,21 & 42,88 & 39,81 & 21,32 \\
\hline & \multicolumn{6}{|c|}{ Efeitos Principais } \\
\hline \multicolumn{7}{|l|}{ Tempo } \\
\hline $144 \mathrm{~h}$ & 28,28 & 26,02 & 40,17 & 8,01 & 9,74 & 13,01 \\
\hline $288 \mathrm{~h}$ & 27,64 & 26,18 & 40,28 & 8,14 & 10,04 & 12,91 \\
\hline \multicolumn{7}{|l|}{ Processo } \\
\hline Com lavagem & 27,83 & 26,14 & 40,31 & 7,78 & 9,76 & 13,13 \\
\hline Sem lavagem & 28,08 & 26,06 & 40,14 & 8,35 & 10,03 & 12,78 \\
\hline & \multicolumn{6}{|c|}{ Probabilidade } \\
\hline Tempo (T) & 0,7656 & 0,8664 & 0,9095 & 0,8316 & 0,6705 & 0,8345 \\
\hline Processo $(\mathrm{P})$ & 0,9067 & 0,9313 & 0,8632 & 0,3690 & 0,7035 & 0,4759 \\
\hline $\mathrm{T} \times \mathrm{P}$ & 0,4981 & 0,5768 & 0,5057 & 0,9244 & 0,9306 & 0,5284 \\
\hline
\end{tabular}

144SL - $144 \mathrm{~h}$ de incubação "in situ" sem lavagem do material; $144 \mathrm{CL}$ - 144h de incubação com lavagem do material em intervalos de $72 \mathrm{~h}$; 288SL - $144 \mathrm{~h}$ de incubação "in situ" sem lavagem do material; $288 \mathrm{CL}$ - 144h de incubação com lavagem do material em intervalos de $72 \mathrm{~h}$.

FDNi - Fibra em detergente neutro indigestível; FDAi - Fibra em detergente ácido indigestível.

CV - Coeficiente de variação.

Fonte: MORAIS et al. (2005).

A avaliação dos marcadores internos FDN e FDA incubados in situ (is) e in vitro (iv) e óxido crômico realizada por FREITAS et al. (2002), indicou que o FDAiv, FDAis e óxido crômico podem ser utilizados na estimativa da produção fecal, sendo que, o FDAiv, por ser obtido de forma mais simples e econômica, tem seu uso recomendado como marcador em ensaios de digestibilidade. Já BERCHIELLI et al. (2005), a partir da avaliação de marcadores internos e óxido crômico em diferentes volumosos, concluíram que, pela alta variabilidade de resultados obtidos, observa-se, possivelmente, a existência de um marcador adequado para cada volumoso utilizado. OLIVEIRA Jr. et al. (2004) levanta ainda outra questão relativa a composição 
Aplicação de técnicas para estudos de ingestão, composição da dieta e digestibilidade

da dieta fornecida aos animais. Os autores observaram que em dietas com alta proporção de concentrado o indicador interno FDNi incubado por 144 horas in vitro não representou a fração indigestível da dieta, sugerindo a necessidade de mais informações acerca de sua recuperação para viabilizar seu uso.

A literatura apresenta tempos de incubação no rúmen (in situ) ou in vitro bastante variados para obtenção das frações indigestíveis, variando entre 72 e 144 horas (BERCHIELLI et al., 2000), 96 horas (RUIZ et al., 2001), 192 horas (ZEOULA et al., 2002) e 288 horas (HUHTANEN et al., 1994), indicando falta de padronização na obtenção dos dados (tempo de incubação e procedimentos laboratoriais). No entanto, o trabalho realizado por MORAIS et al. (2005), avaliando os tempos 144 e 288 horas de incubação in situ para obtenção da FDN e FDA indigestíveis, não demonstrou diferença entre o teor dos indicadores (TABELA 2) e estimativas de produção fecal e fluxo da digesta duodenal (TABELA3) para os diferentes tempos considerados.

Outro aspecto abordado na avaliação de MORAIS et al. (2005), é a possibilidade de, pelo alto tempo de permanência dos sacos no rúmen, haver obstrução dos poros pela deposição de matéria degradada ou microrganismos, gerando, assim, a necessidade de limpeza periódica dos sacos de náilon para que a degradação das amostras não seja comprometida. Entretanto, o processo de retirada dos sacos do rúmen para lavagem também não causou alteração nos parâmetros avaliados (TABELAS 2 e 3).

TABELA 3 - FLUXO DA DIGESTA DUODENAL E PRODUÇÃO FECAL ESTIMADOS POR MEIO DE INDICADORES INTERNOS.

\begin{tabular}{|c|c|c|c|c|}
\hline \multirow{3}{*}{ Tratamentos } & \multicolumn{4}{|c|}{ Parâmetros } \\
\hline & \multicolumn{2}{|c|}{ Fluxo digesta duodenal $(\mathrm{kg} / \mathrm{d})$} & \multicolumn{2}{|c|}{ Produção fecal (kg/d) } \\
\hline & FDNi & FDAi & FDNi & FDAi \\
\hline 144SL & 4,80 & 3,86 & 3,05 & 2,75 \\
\hline $144 \mathrm{CL}$ & 4,54 & 3,77 & 2,92 & 2,62 \\
\hline 288SL & 4,57 & 3,91 & 2,87 & 2,89 \\
\hline 288CL & 4,55 & 3,71 & 2,93 & 2,54 \\
\hline \multirow[t]{2}{*}{ CV (\%) } & 30,34 & 38,47 & 29,48 & 31,54 \\
\hline & \multicolumn{4}{|c|}{ Efeitos Principais } \\
\hline \multicolumn{5}{|l|}{ Tempo } \\
\hline $144 \mathrm{~h}$ & 4,67 & 3,81 & 2,99 & 2,68 \\
\hline $288 \mathrm{~h}$ & 4,56 & 3,81 & 2,90 & 2,71 \\
\hline \multicolumn{5}{|l|}{ Processo } \\
\hline Com lavagem & 4,54 & 3,74 & 2,93 & 2,58 \\
\hline \multirow[t]{2}{*}{ Sem lavagem } & 4,69 & 3,88 & 2,96 & 2,82 \\
\hline & \multicolumn{4}{|c|}{ Probabilidade } \\
\hline Tempo (T) & 0,6629 & 0,9811 & 0,5813 & 0,8430 \\
\hline Processo (P) & 0,5653 & 0,5753 & 0,8230 & 0,1079 \\
\hline $\mathrm{T} \times \mathrm{P}$ & 0,6249 & 0,8476 & 0,5257 & 0,4651 \\
\hline
\end{tabular}

144SL - $144 \mathrm{~h}$ de incubação sem lavagem do material; $144 \mathrm{CL}$ - 144h de incubação com lavagem do material em intervalos de 72 h; $288 S L$ - 144 h de incubação sem lavagem do material; $288 \mathrm{CL}$ - $144 \mathrm{~h}$ de incubação com lavagem do material em intervalos de $72 \mathrm{~h}$.

FDNi - Fibra em detergente neutro indigestível; FDAi - Fibra em detergente ácido indigestível.

CV - Coeficiente de variação.

Fonte: MORAIS et al. (2005). 
Em relação a avaliação de marcadores para estimativa de fluxo da digesta, AHVENJÄRVI et al. (2003) abordam a questão da necessidade de utilização concomitante de mais de um marcador, marcando as fases líquidas e sólidas distintamente, considerando ainda que para a fase sólida pode ocorrer a necessidade de marcadores para partículas da digesta de diferentes tamanhos. Essa questão é também abordada por BERCHIELLI (2003), ao considerar que as distintas fases ou partículas possuem diferentes taxa de passagem, podendo resultar, dessa forma, em coleta de digesta duodenal ou omasal que não seja representativa da amostra real. Com base nessas informações, é indicado o sistema de duplo ou triplo marcadores, dependendo de técnica que possibilite a separação da digesta em duas fases (líquida e sólida) ou três fases (líquida, partículas pequenas e grandes), respectivamente.

Mesmo em casos onde não há registro do dado de ingestão é possível determinar a digestibilidade pela relação entre a concentração do indicador no alimento e fezes. Pode-se considerar que ao administrar um indicador, se a recuperação nas fezes for total, a quantidade de matéria seca ingerida $(I)$ vezes a concentração no indicador $\left(C_{\|}\right)$será igual à produção da matéria seca das fezes $(E)$ vezes sua concentração no indicador $\left(C_{E}\right)$ :

$$
I x C_{1}=C x C_{E}
$$

sendo,

$$
E / I=C_{I} / C_{E}
$$

A digestibilidade, portanto, pode ser calculada como:

$$
D=1-C_{I} / C_{E}
$$

Da mesma forma, a digestibilidade de qualquer fração da matéria seca $(D n)$ pode ser calculada como:

$$
D_{n}=1-\frac{C_{I}}{C_{E}} \times \frac{n_{e}}{n_{i}}
$$

Sendo $n_{e}$ e $n_{i}$ as concentrações do nutriente considerado nas fezes e no alimento, respectivamente.

Métodos in situ: É uma técnica bastante utilizada para estimar a degradação ruminal de nutrientes pela relativa simplicidade e baixo custo quando comparada aos experimentos in vivo.

A técnica consiste em incubação no rúmen de sacos de náilon contendo os alimentos a serem avaliados e a medida do desaparecimento da matéria seca e nutientes em intervalos de tempo pré-determinados, apresentando como vantagem o fato do processo de degradação ocorrer em condições reais do rúmen.

A utilização da técnica in situ é mais empregada para determinação da degradação protéica no rúmen, embora seu uso também venha sendo destinado a estimativas da degradação da matéria seca e carboidratos. Um dos erros associados a esta metodologia é a premissa de que toda proteína solubilizada é completa e instantaneamente degradada no rúmen (MAHADEVAN et al, 1980), no entanto, em alguns casos, a taxa de degradação para a fração solúvel pode ser menor do que para a fração insolúvel.

A contaminação do resíduo da fermentação resultante da colonização das partículas é uma considerável fonte de erro nas estimativas da extensão e taxa de degradação da proteína no rúmen, especialmente em alimentos ricos em proteína; havendo ainda correlação positiva entre tempo de incubação e contaminação (WANDERLEY et al., 1993).

VANZANT et al. (1998) citam que alguns marcadores como ${ }^{15} \mathrm{~N}$ e purinas vêm sendo empregados com o propósito de isolar quanto da proteína determinada no resíduo é oriunda de microrganismos. No entanto, segundo o mesmo autor, escasso número de trabalhos na literatura fazem menção a algum tipo de correção decorrente de contaminação microbiana efetuada nas determinações de degradabilidade de matéria seca, matéria orgânica ou proteína bruta.

Já em relação aos parâmetros de degradação da porção fibra, o problema de contaminação microbiana é reduzido. MASS et 
Aplicação de técnicas para estudos de ingestão, composição da dieta e digestibilidade

al. (1999) cita que a lavagem do resíduo de degradação com solução de detergente neutro possui a propriedade de remover os microrganismos aderidos as partículas.

Um outro aspecto negativo relacionado à utilização da metodologia é a necessidade de um grande número de horários de incubação ruminal. OLAISEN et al. (2003), entretanto, demonstraram que a utilização de reduzido número de horários (dois ou três horários) é eficiente na determinação dos parâmetros de degradação através de modelos de regressão bilineares quando comparados aos parâmetros determinados com a utilização de maior número de tempos de incubação (sete ou oito horários). CARMO et al. (2001) avaliaram a utilização de diferentes números de horários de incubação para cana-de-açúcar e apresentaram que os tempos 3 e 6 horas não diferiram entre si, sendo o mesmo comportamento observado entre horários 72, 96 e 120 horas. Os autores sugerem que sejam excluídos os tempos 3, 96 e 120 horas, aumentando os horários de incubação entre os horários 6 e 12 horas. O maior número de horários, nas primeiras horas de incubação, pode ser efetivo na obtenção de melhores estimativas do comportamento de degradação em alimentos que são rapidamente degradados no rúmen.

A digestibilidade intestinal da proteína pode ser obtida através da metodologia in situ, conhecida como técnica dos sacos móveis. A técnica consiste em introduzir no duodeno sacos de náilon contendo pequena quantidade de amostra previamente incubada no rúmen, sendo estas recuperadas normalmente nas fezes. No entanto, a digestibilidade intestinal da proteína não degradável no rúmen (PNDR) é muito variável. Alimentos concentrados ricos em proteína apresentaram maior digestibilidade da PNDR em relação a alimentos com maior proporção de fibras, sendo esta digestibilidade afetada possivelmente por frações outras que não o nitrogênio (VANHATALO e KETOJA, 1995).

Método in vitro: A metodologia descrita por TILLEY e TERRY (1963) ainda é a mais utilizada para predição da digestibilidade in vitro, simulando a digestão ruminal por 48 horas, seguida por digestão com pespsina e ácido fraco $(\mathrm{pH} 2,00)$ por 48 horas. Neste caso, o resíduo indigestível engloba microrganismos e outros compostos insolúveis em pepsina.

A confiabilidade na obtenção dos dados é dependente de alguns fatores, como diluição do fluido ruminal, manutenção das condições do rúmen, tipo de tamponante utilizado, tamanho da partícula incubada e dieta que o animal doador do conteúdo ruminal estava recebendo (STERN et al., 1997).

De acordo com PEREZ (1997) os sistemas in vitro não reproduzem com total fidelidade os resultados obtidos in vivo, podendo ser acoplado ou não a sistemas de equações de regressão, onde a capacidade de predição é resultante da similaridade entre a técnica e o processo digestivo do ruminante.

A técnica de produção de gás é uma outra possibilidade para estudos que envolvem taxa e extensão da degradação dos alimentos. $O$ uso desta metodologia na determinação da digestão de carboidratos apresenta vantagens em relação ao método tradicional por considerar tanto a fração solúvel como a insolúvel do alimento (PELL e SCHOFIELD, 1993), sendo apresentado pelos autores alta correlação entre a produção de gás e desaparecimento do FDN $\left(r^{2}=0,99\right)$ e matéria seca $\left(r^{2}=0,95\right)$.

VAREL e KREIKEMEIR (1995) traçaram uma comparação entre as metodologias in vitro e in situ e observaram diferenças nos resultados obtidos para tempo de colonização das amostras pelos microrganismos, taxa e extensão de digestão. Os autores atribuem as diferenças a menor concentração microbiana presente na técnica in vitro quando comparada ao rúmen do animal, sendo ainda que as tentativas com intuito de aumentar a concentração microbiana não obtiveram sucesso, uma vez que ocorre rápido acúmulo dos produtos finais oriundos da fermentação e conseqüente queda no $\mathrm{pH}$.

A redução do $\mathrm{pH}$ no inoculo é um dos maiores problemas apresentados pela técnica in vitro podendo afetar a digestibilidade da fibra, uma vez que os microrganismos celulolíticos são os mais sensíveis a queda do $\mathrm{pH}$ (STERN et al., 1997). 


\section{Considerações Finais}

A literatura apresenta uma grande amplitude de técnicas para determinação de parâmetros importantes na pesquisa animal. No entanto, alguns critérios devem ser adotados na escolha da técnica empregada. Os recursos disponíveis, financeiros e operacionais, a possibilidade de implantação da técnica e a busca por maior precisão das estimativas devem nortear a seleção das metodologias a serem utilizadas. O emprego de indicadores é uma alternativa bastante difundida pela grande variabilidade de opções e, dependendo do indicador, facilidade de uso e baixo custo, possibilitando a estimativa de consumo e digestibilidade nos diferentes compartimentos gastrintestinais. Os alcanos têm surgido como opção para estimativa dos parâmetros considerados, apresentando resultados bastante satisfatórios.

\section{REFERÊNCIAS}

AHVENJÄRVI, S.; VANHATALO, A.; SHINGFIELD, K.J., HUHTANEN, P. Determination of digesta flow entering the omasal canal of dairy cows using different marker systems. British Journal of Nutrition, London, v.90, n.1, p.41-52, 2003.

ALLEN, M. S. Effects of diet on short-term regulation of feed intake by lactating dairy cattle. Journal of Dairy Science, Savoy, v.83, n.7, p.1598-1624, 2000.

BERCHIELLI, T.T. Uso de marcadores em estudos de ingestão, digestibilidade, composição da dieta, trânsito e fluxo digestivo. Jaboticabal: Faculdade de Ciências Agrárias e Veterinárias/ UNESP, 2003. 264p. Tese (Livre Docência) Faculdade de Ciências Agrárias e Veterinárias/ UNESP, 2003.

BERCHIELLI, T.T.; ANDRADE, P.; FURLAN, C. L. Avaliação de indicadores internos em ensaios de digestibilidade. Revista Brasileira de Zootecnia, Viçosa, v.29, n.3, p.830-833, 2000.

BERCHIELLI, T.T.; OLIVEIRA, S.G.; CARRILHO, E.N.V.M.; FEITOSA, W.; LOPES, A.D. Comparação de marcadores para estimativas de produção fecal e de fluxo de digesta em bovinos. Revista Brasileira de Zootecnia, Viçosa, v.34, n.3, p.986-995, 2005.
CARMO, C.A.; BERCHIELLI, T.T.; ANDRADE, P.; ZEOLA, N.M.B.L. Degradabilidade da matéria seca e fibra em detergente neutro da cana-de-açúcar-deaçúcar (Saccharum spp) com diferentes fontes de proteína. Revista Brasileira de Zootecnia, Viçosa, v.30, n.6, p.2126-2133, 2001 (Suplemento).

COCHRAN, R.C.; ADAMS, D.C.; WALLACE, J.D. Predicting digestibility of different diets with internal markers: evaluation of four potential markers. Journal of Animal Science, Savoy, v.63, n. 5, p. 1476-1483, 1986.

DOVE, H.; MAYES, R. W. The use of plant wax alkanes as marker substances in studies of the nutrition of herbivores: a review. Australian Journal of Agricultural Research, Collingwood, v.42, n.6, p. 913-952, 1991.

FREITAS, D.; BERCHIELLI, T.T.; SILVEIRA, R.N. Produção fecal e fluxo duodenal de matéria seca e matéria orgânica estimados por meio de marcadores. Revista Brasileira de Zootecnia, Viçosa, v.31, n.3, p.1521-1530, 2002 (Suplemento).

HOVELL, F.D.B.; NGAMBI, J.W.W.; BARBER, W.P. The voluntary intake of hay by sheep in relation to its degradability in the rumen as measured in nylon bags. Animal Production, Lincoln, v.42, n.1, p.111-118, 1986.

HUHTANEN, P.; KAUSTELL, K.; JAAKKOLA, S. The use of internal markers to predict total digestibility and duodenal flow of nutrients in cattle given six different diets. Animal Feed Science and Technology, Amsterdam, v.48, n.1, p.211-227, 1994.

ÍTAVO, L.C.V.; VALADARES FILHO, S.C.; SILVA, F.F.; VALADARES, R.F.D.; CECON, P.R. ÍTAVO, C.C.B.F.; MORAES, E.H.B.K.; PAULINO, P.V.R. Consumo, degradabilidade ruminal e digestibilidade aparente de fenos de gramíneas do gênero Cynodon e rações concentradas utilizando indicadores internos. Revista Brasileira de Zootecnia, Viçosa, v.31, n.2, p.1024-1032, 2002 (Suplemento).

LASCANO, C.E.; BOREL, R.; QUIROZ, R. Recomendaciones sobre metodologia para la medicion de consumo y digestibilidad in vivo. In: RUIZ,M.; RUIZ, A. (Eds.) Nutrición de rumiantes. Guia metodológico de investigación. Costa Rica: ALPA Rispal, 1990, p.159-168.

MAHADEVAN, S.; ERFLE, J.D.; SAUER, F.D. Degradation of soluble and insoluble proteins by Bacteroides amylophilus protease and by rumen microrganism. Journal of Animal Science, Savoy, v.50, n.6, p.723-728, 1980. 
Aplicação de técnicas para estudos de ingestão, composição da dieta e digestibilidade

MALAFAIA, P.A.M.; VALADARES FILHO, S.C.; SILVA, J.F.C. Degradabilidade potencial de alguns volumosos estimada in situ e in vitro. In: REUNIÃO ANUAL DA SOCIEDADE BRASILEIRA DE ZOOTECNIA, 33., 1996, Fortaleza. Anais... Fortaleza: SBZ, 1996. p.312.

MASS, R.A.; LARDY, G.P.; GRANT, R.J.; KLOPFENSTEI, T.J. In situ neutral detergent insoluble nitrogen as a method for measuring forage protein degradability. Journal of Animal Science, Savoy, v.77, n.7, p.1565-1571, 1999.

MAYES, R. W; LAMB, C. S.; COLGROVE, P. M. The use of dosed and herbage $n$-alcanes as markers for the determination of herbage intake. Journal of Agricultural Science, Cambridge, v.107, n.1, p.161170, 1986.

MAYES, R.W.; DOVE, H.; CHEN, X.B. Advances in the use of faecal and urinary markers for measuring diet composition, herbage intake and nutrient utilization in herbivores. In: JOURNET, M.; GRENET, E.; FARCE, M.H. et al. (Eds.) Recent developments in the nutrition of herbivores. INRA editions, 1995. p.381-406.

MAYES, R.W.; DOVE, H. Measurement of dietary nutrient intake in free-ranging mammalian herbivores. Nutrition Research Reviews, London, v.13, n.2, p.107-138, 2000.

MERTENS D.R. Regulation of forage intake. In: FAHEY Jr., D.C. Forage quality, evaluation and utilization. Madison: American Society of Agronomy.1994. p.450-492.

MINSON, D. J. Forage in ruminant nutrition. San Diego, California: Academic Press, Inc., 1990. 483 p.

MORAIS, J.A.S.; OLIVEIRA, S.G.; TOROVELÁSQUEZ, P.A.; RIVERA-RIVERA, A.; BERCHIELLI. T.T. Avaliação de metodologias na determinação de indicadores internos para estimar o fluxo da digesta duodenal e a produção fecal em bovinos. In: REUNIÃO ANUAL DA SOCIEDADE BRASILEIRA DE ZOOTECNIA, 42, 2005, Goiânia. Anais ... Goiânia: SBZ/technoMEDIA, 2005. CDROM.

NOCEK, J.E. In situ and other methods to estimate protein and energy digestibility. A review. Journal of Dairy Science, Savoy, v.71, n.8, p.2051-2069, 1988.

OLAISEN, V.; MEJDELL, T.; VOLDEN, H.;NESSE, N. Simplified in situ method for estimating ruminal dry matter and protein degradability of concentrates. Journal of Animal Science, Savoy, v.81, n.3, p.520528, 2003.
OLIVEIRA JR., R.C.; PIRES, A.V.; FERNANDES, J.J.R.; SUSIN, I.; SANTOS, F.A.P., NASCIMENTO FILHO, V.F.; ARAÚJO, R.C. Avaliação de marcadores para estimar a digestilidade dos nutrientes em novilhos nelore alimentados com dietas contendo alto teor de concentrado e fontes nitrogenadas. Revista Brasileira de Zootecnia, Viçosa, v.33, n.3, p.749-758, 2004.

PELL, A.N.; SCHOFIELD, P. Computerized monitoring of gas production to measure forage digestion in vitro. Journal of Dairy Science, Savoy, v.76, n.6, p.1063-1073, 1993.

PEREZ, J. R. Sistemas para a estimativa de digestibilidade in vitro. In: SIMPÓsIO INTERNACIONAL DE DIGESTIBILIDADE EM RUMINANTES, 1997, Lavras, Anais... Lavras: UFLA-FAEPE, 1997. p.55-68.

POND, K. R.; ELLIS, W.C.; MATIS, J.H. Passage of chromium-mordanted and rare earth-labeled fiber: time dosing kinetics. Journal of Animal Science, Savoy, v. 67, n.4, p. 1020-1028, 1989.

POPPI, D.P.; FRANCE, J.; MCLENNAN, S.R. Intake, Passage and Digestibility. In: THEODOROU, M.K.; FRANCE, J. Feeding systems and feed evaluation models. New York: CABI, 2000. p.35-52.

RUIZ, R.; VAN SOEST, P.J.; VAN AMBURGH, M.E.; FOX, D.G.; ROBERTSON, J.B. Use of chromium mordanted neutral detergent residue as a predictor of fecal output to estimate intake in grazing high producing Holstein cows. Animal Feed Science and Technology, Amsterdam, v.89, n.3-4, p.155-164, 2001.

STERN, M.D.; BACH, A.; CALSAMIGLIA, S. Alternative techniques for measuring nutrient digestion in ruminants. Journal of Animal Science, Savoy, v.75, n.12, p.2256-2276, 1997.

TILLEY, J. M. A.; TERRY, R. A. A two stages technique for the "in vitro" digestion of forage crops. Journal of British Grassland Society, London, v.18, n.2, p.104-111,1963.

TITGEMEYER, E.C. Design and interpretation of nutrient digestion studies. Journal of Animal Science, Savoy, v.75, n.8, p.22352247, 1997.

VANHATALO, A.; KETOJA, E. The role of the large intestine in post-ruminal digestion of feeds as measured by the mobile-bag method in cattle. British Journal of Nutrition, London, v.73, n.4, p.491-505, 1995. 
VANZANT, E.S.; COCHRAN, R.C.; TITGEMEYER, E.C. Standardization of in situ techniques for ruminant feedstuff evaluation. Journal of Animal Science, Savoy, v.76, n.10, p.2717-2729, 1998.

VAN SOEST, P.J. Symposium on factors influencing the voluntary intake of herbage by ruminants: Voluntary intake relation to chemical composition and digestibility. Journal of Animal Science, Savoy, v.24, n.3, p.834-844, 1965.

VAN SOEST, P.J. Nutritional ecology of the ruminant. 2. ed. Ithaca: Cornell University Press, 1994. $476 \mathrm{p}$.

VAREL, V.H.; KREIKEMEIER, K.K. Technical note: Comparison of in vitro and in situ digestibility methods. Journal of Animal Science, Savoy, v.73, n.2, p.578-582, 1995.

Recebido para publicação: 25/03/2005

Aprovado:

$28 / 08 / 2005$
WANDERLEY, R.C.; HUBER, J.T.; WU, Z.; PESSARAKLI, M.; FONTES, C. Influence of microbial colonization of feed particle on determination of nitrogen degradability by in situ incubation. Journal of Animal Science, Savoy, v.71, n.12, p.3073-3077, 1993.

ZEOULA, L.M.; KASSIES, M.P.; FREGADOLLI, F.L. Uso de indicadores na determinação da digestibilidade parcial e total em bovinos. Acta Scientiarum, Maringá, v.22, n.3, p.771-777, 2000.

ZEOULA, L.M.; PRADO, I.N.; DIAN, P.H.M.; GERON, L.J.V.; CALDAS NETO, S.F; MAEDA, E.M.; PERÓN, P.D.P.; MARQUES, J.A.; Falcão, A.J.S. Recuperação fecal de indicadores internos avaliados em ruminantes. Revista Brasileira de Zootecnia, Viçosa, v.31, n.4, p.1865-1874, 2002. 